EACH ISSUE of The Annals of Iowa brings to light the deeds, misdeeds, and accomplishments of our predecessors and shows how they fit into the intricate mosaic of Iowa's past. Its in-depth articles will satisfy even the most serious explorer of Iowa's past.

Anyone with a serious interest in Iowa history will gain valuable perspective from the pages of the Annals. Give it as a gift to a friend or relative. Check to see if your public, school, or academic library subscribes; if they don't, encourage them to do so or, better yet, donate a subscription.

8

$\square$ Annals of Iowa Subscription $\quad \square$ New $\square$ Renewal $\square$ Gift* $\square$ One year, $\$ 24.95$

$\square$ Two years, $\$ 44.95$

$\square$ Three years, $\$ 64.95$

Name

Address

City State Zip

Make check payable to the State Historical Society of Iowa and return with this coupon (or a photocopy of it) to:

Subscriptions

State Historical Society of Iowa

402 Iowa Avenue

Iowa City IA 52240

$\square$ Please send information on membership in the State Historical Society of Iowa.

*For gift subscriptions, write the recipient's name and address on this form, and include your name and address on the back or on a separate sheet of paper. Also indicate how you would like your gift card signed. 


\section{Index to Volume 76}

Abbott, Harry, 431

Abbott, Theophilus, 200

Abolitionizing Missouri: German Immigrants and Racial Ideology in Nineteenth-Century America, reviewed, 347-48

Adams, Henry, book by, reviewed, 146-48

Adcock, Homer, 27

AFL-CIO merger, 103-4

African Americans, 164-65, 178-79; soldiers, 403-4; women, 113

Agribusiness, 61, 66-69

Agricultural colleges, 180-208

Agricultural Experiment Station, Iowa State University, 56, 58

Agricultural Society, Iowa State, 191-92

Agricultural transformation, 48-50, $52,60-61,64$

Alcalá, Charles, 423

Alcalá, Lawrence, 421-22, 424, 430

Alcalá, Thelma, 421-22, 430

Alcohol, as fuel, 57-58, 61

Alcohol use, 393

Alcoholism, 11-12

Aldrich, Charles, 189

Alexander, Benjamin F., book by, reviewed, 128-29

All the Wild that Remains: Edward Abbey, Wallace Stegner, and the American West, reviewed, 372-74

Alling, Calvin P., 172

Alvarado, Trinidad, 419

Amana Colonies, 1932-1945, reviewed, 245-46

Amato, Joseph A., book by, reviewed, 464-65

American Antiquities: Revisiting the Origins of American Archaeology, reviewed, 217-20
American Guides: The Federal Writers' Project and the Casting of American Culture, reviewed, 451-53

American Serengeti: The Last Big Animals of the Great Plains, reviewed, 114-15

Anamosa, Iowa, 177

Ancestral Mounds: Vitality and Volatility of Native America, reviewed, 217-20

Anderson, Douglas Firth, book review by, 222-24

Anderson, Dwight, 89

Anderson, Kristen Layne, book by, reviewed, 347-48

Anderson, William, book edited by, reviewed, 131-32

Andreasen, Bryon C., book review by, 119-21

Ankeny, Iowa, 59

Antoine, Mary Elise, book by, reviewed, 339-40

Apps, Jerry, book by, reviewed, 459

Army, U.S., 381-405

Army of the Southwest, Civil War, $161-76,178-79$

Ashby, Dorothy, 386, 393

Athletics, 397-402

Aubrey, Dean, 83, 85, 99, 108

Ayres, Homer, 90

Babel Proclamation, 313

Bains, David R., book review by, 368-70

Baker, Newton, 384

Baker, Thomas R., book by, reviewed, 350-52

Banks and banking, 176-77

Bankson, Gene, 83

Bankson, Virgil, 83

Barker-Devine, Jenny, book review by, 358-61 
Barloon, Mark, book review by, 22829

Barnhardt, Terry A., book by, reviewed, 217-20

Baseball, 397, 398

The Baseball Whisperer: A Small-Town Coach Who Shaped Big League

Dreams, reviewed, 460-61

Basketball, 398

Bayor, Ronald H., book edited by, reviewed, 250-52

Beardsley, Harry, 19

Beecher, Henry Ward, 167

Beilein, Joseph M., Jr., book by, reviewed, 349-50

Belknap, Kitturah P., 264

Beneke, Donald, 40, 44

Benn, David W., book review by, 337-38

Bennett, Clifford, 26

Bennett, Eric, book by, reviewed, 150-52

Benson, Ezra Taft, 107

Berlage, Nancy K.: book by, reviewed, 358-61; book review by, 361-63

Bernard, Iowa, 29

Bertrand, Dorothy, 411

Bettendorf Car Co., 422

Better Trucking Bureau, Iowa, 10

The Big Marsh: The Story of a Lost Landscape, reviewed, 209-15

Bigham, Darrel, book review by, 347-48

Biles, Roger, book review by, 141-42

Birk, Megan, book by, reviewed, 241-43

Bishop, Lester, 82

Black Hawk Labor Council, AFLCIO, 106, 112-13

Bleyart, Tamiel, 26

Blue, Robert D., 63, 70, 86

Bohemian Catholics, 301, 303-7, 310

Boies, Horace, 5

Bonds of Union: Religion, Race, and Politics in a Civil War Borderland, reviewed, 121-24

Boone, Iowa, 280

The Borderland of Fear: Vincennes, Prophetstown, and the Invasion of the Miami Homeland, reviewed, 435-37

Boszhardt, Robert F. See Schrab, Geri
Bottiger, Patrick, book by, reviewed, 435-37

Bowes, John P.: book by, reviewed, 220-22, book review by, 118-19

Boxing, 401

Brannan Plan, 94

Brant, Irving N., 386

Bremer, Jeff, article by, 261-89

Brettell, Caroline, book by, reviewed, 124-26

Brewer, William, 194

Brewer-Bonebright, Sarah, 274, 286

Brewster, Charles, 279

Brighton, Iowa, 281

Bruner, Robert, 27

Buchanan, James, 75

Buchanan, Robert, 57-58

Buecker, Thomas R., book by, reviewed, 236-38

Buenker, John D., book review by, 250-52

Buggeln, Gretchen, book by, reviewed, 368-70

Bull, Russell, 95

Bureau of Criminal Investigation, Iowa, 25, 39

Burlington, Iowa, 59, 295, 296

Burlington Hawkeye, 23

Burnes, Bishop James J., 35

Bushnell, Alexander, 300-301

Bushwhackers: Guerrilla Warfare, Manhood, and the Household in Civil War Missouri, reviewed, 349-50

Butler, Benjamin, 164-65

Butts, Albert, 277

Buxton, William, 287

Caddock, Earl, 401-2

Call, George S., 53

Camp, John, 31

Camp Dodge, 381-405

Camp Dodger, 381-405

Camp Funston, 398-99, 401

Canby, Edward, 178

Canning industry, 67-68

Cannon, Brian Q., book review by, 143-44

Cano, Elena, 426, 427, 431

Cano, Magdaleno, 422, 423, 424, 42631; photo of, 428

Cano, María, 422, 423, 426-31 
Cano, Vincent, 426, 428, 429-30, 432

Cantwell, Christopher D., et al., book edited by, reviewed, 145-46

Carmichael, Sarah E., 286

Carpenter, John Alden, 396

Carr, B. O., 165, 166

Carroll, George, 279

Carroll, Iowa, 27

Carter, Heath W. See Cantwell, Christopher D.

Carter Lake, Iowa, 28, 30

Catholics, Roman, 35, 290-315

Cedar Rapids, Iowa, 25-26, 27

Cedar Rapids Gazette, 20

Centerville, Iowa, 59

Centerville Iowegian, 54

Chamberlin, James, 284

Champ, Orvel, 79, 82, 84, 85

Charles City, Iowa, 89

Chase, Rachelle, book by, reviewed, 448-49

Chicago, 176

Chicago, Rock Island and Pacific Railroad Co., 406-32

Childers, Leisl Carr, book review by, 357-58

Christie, Mary Parsons, 269-70

Christie, Robert, 268

Church, Frank, 29

Churches, 17-18, 34-36, 290-315

City-Manager plan, Ottumwa, 81

Civil War, 161-79, 187-88

Clark, Dick, 112

Clark, Joseph, 82

Clements, Kendrick A., book review by, 363-66

Clinton, Iowa, 26, 27, 30

Clough, Samuel, 265

Cohagan, Thomas, 99

Cohen, Sheila Terman, book by, reviewed, 352-54

Commerce Commission, Iowa State, 11

Conard, Rebecca, review essay by, 209-15

Confiscation Act, Second, 164

Congress of Industrial Organizations (CIO), 71-113

Conner, Robert, 108

Contraband, Civil War, 161-79

Cook, R. H., 386
Coon, Stephen C., book review by, 455-56

Cooney, John, 106, 112

Cooper, Lyle, 83

Cordery, Simon, book by, reviewed, 135-37

Cordery, Stacy A., book review by, 232-33

Corn-based alcohol, 57-58, 61

Cornell, Ezra, 193

Cotton trade, 169-72

Couch, A. A., 53, 58

Couch, Julianne: book by, reviewed, 254-56; book review by, 463-64

Council Bluffs, Iowa, 26, 271-72, 283

Council of Churches, Iowa, 17, 34

Court of Inquiry on the Sale of Cotton and Produce (1863), 168-72, 178

Cowie, Jefferson, book by, reviewed, 141-42

Cowper, Dean Holmes, 395-96

Coxey's Army: Popular Protest in the Gilded Age, reviewed, 128-29

"Creating a Barrio in Iowa City, 19161936: Mexican Section Laborers and the Chicago, Rock Island and Pacific Railroad Company," 406-32

Cresco, Iowa, 29

Cronin, Frank, 79

Crop prices, 270, 272

Crowders, Mary Lacey, 273

Crusading Iowa Journalist Verne Marshall: Exposing Graft and the 1936 Pulitzer Prize, reviewed, 453-55

Culver, John, 112

Cumo, Christopher, book review by, 354-55

Curran, Leigh, 40

Curricula, higher education, 195-96, 198, 202-4, 206-7

Currier, L. C., 396

Curtis, Henry Z., 176

Curtis, Samuel Ryan, 161-70, 178

Czech Catholics, 301, 303-7, 310

Dale, Vernon, 92-95, 101-4

Daniels, Addison, 279

Davenport, Iowa, 26, 28, 29-31, 283, 295, 411

Davenport Democrat, 19, 24

Deacon, Elma, 29 
Defense industries, 56-62

Delton, Jennifer, book review by, 462-63

Democratic Party, 21, 72-73, 77, 84, 107-8, 111-13

Depression, Great, 422

Deslippe, Dennis, book review by, 246-48

Des Moines, Iowa, 27, 276, 280, 385, 390, 391, 402, 423

Des Moines Architecture and Design, reviewed, 149-50

Des Moines Register, 8-9, 19, 25

Des Moines River, 276

Development Commission, Iowa, 47, 63-70

De Witt, Simeon, 183

Dexter, Freeland, book by, reviewed, 445-46

Dictionary of Midwestern Literature, volume 2, Dimensions of the Midwestern Literary Imagination, reviewed, 433-35

Dietz, Riley, 37

Dinan, John, book review by, 115-18

Dirck, Brian, book review by, 230-32

Disease, sexually transmitted, 383-84

Dobbs, Farrell, 79

Drake, Janine Giordano. See Cantwell, Christopher D.

Drake Relays, 397, 399

Drake University, 395, 397

Dubuque, Iowa, 26, 28, 58, 75, 27980, 281

Duffield, George C., 273, 286

Eagle Grove, Iowa, 56, 57, 58

Economic development, 47-70

Edmonds, Michael, book review by, 451-53

Education: higher, 180-208; science, 184

Effland, Anne, book review by, 24143

Eighteenth Amendment to the U.S. Constitution, 6, 7

88th Infantry Division, 381-405

Ellmaker, Reuben, 267, 277-78

Ely, John, 34

Emancipation, 167

Emigrants, overland, 270
Emonds, William, 304, 305, 309, 312

Engineering Victory: The Union Siege of Vicksburg, reviewed, 228-29

Ensley, Bishop R. Gerald, 34

Episcopalians, 34-35

Erbe, Norman, 2, 13-21, 23, 32, 110; photo of, 14

Espy, G. R., store, 282

Etcheson, Nicole, book review by, 121-24

Etherton, M. H., 26

Ethnicity, 290-315

Ethyl alcohol, 57-58, 61

Evangelical Ministers Association, Greater Des Moines, 17

Everhart, Kenneth, 92-95, 102, 104

Everyday Life: How the Ordinary Became Extraordinary, reviewed, 464-65

“Explaining Gubernatorial Stability in Iowa: A Review Essay and Author's Response," 316-34

Fair Employment Practices Act, 111

Fair Employment Practices Commission, 113

Fairall, Laurence R., 381, 385-90, 392-94, 404-5

Fairchild, Ephraim Gard, 268

Fairs, county, 91

Farm Bureau Federation: American, 94; Iowa, 32, 50, 53, 107, 109

Farm-Labor Association, 106

Farm mechanization, 60, 65

Farm Processing Cooperative, Iowa, 58

Farmer-labor organizing, 87, 94, 105-7

Farmers Helping Farmers: The Rise of the Farm and Home Bureaus, 19141935, reviewed, 358-61

Farmers Union: Iowa, 87-91, 105; National, 90

Feis, William B., book review by, 350-52

Fenwick, Bishop Edward, 295

Filliman, Edward, 83, 85, 86, 89, 90, 93, 95, 98-99

Fitzpatrick, Edward, 82

Flores, Dan, book by, reviewed, 11415

Foerstner, Abigail, book review by, 245-46 
Folk, Holly, book review by, 355-57

Following Father Chiniquy: Immigration, Religious Schism, and Social Change in Nineteenth-Century Illinois, reviewed, 124-26

Football, 398-401

Foote, Albert E., 204

Ford, Bridget, book by, reviewed, 121-24

Forsman, Darrel, 67-68

Forsman, Don, 67

Fort Dodge, Iowa, 27, 189, 276

Fort Madison, Iowa, 26, 279, 282

Foster, G. D., 389

Foster, J. Ellen, 4

Fostering on the Farm: Child Placement in the Rural Midwest, reviewed, 241-43

The 4-H Harvest: Sexuality and the State in Rural America, reviewed, 361-63

Free Spirits: Spiritualism, Republicanism, and Radicalism in the Civil War Era, reviewed, 437-39

Friend, I. H., 281

Friends, Iowa Yearly Meeting of, 34

Friley, Charles, 63

From America to Norway: NorwegianAmerican Immigrant Letters, 18381914, volume 3, 1893-1914, reviewed, 446-48

From Hometown to Battlefield in the Civil War: Middle Class Life in Midwest America, reviewed, 224-26

"From Model Farm to a College with Students: Benjamin Gue, Peter Melendy, Adnoijah Welch, and the Iowa Agricultural College and Model Farm, 1867-1869," 180-208

From Our Special Correspondent: Dispatches from the 1875 Black Hills Council at Red Cloud Agency, Nebraska, reviewed, 236-38

Frontier Democracy: Constitutional Conventions in the Old Northwest, reviewed, 115-18

Frontier settlement, 261-89

Fry, John J., book review by, 131-32

Fulton, Jack, 25-26

Gable, Dan, book by, reviewed, 15253
Gardens, 427-28

Garnavillo, John, 266-67

Garrett, J. Tracy, 53

Gavitt, Benjamin, 277

Gelpcke, Theo., 176-77

German Catholics, 290, 294, 297, 299 , $301,302,303,304,305,307,311-15$

Gessner, David, book by, reviewed, 372-74

Gibbons, Mike, 398, 401

Gilbert, Jess, book by, reviewed, 243-45

Gillette, Guy M., 56-58

Glenn, Gene, 108

Godfert, Anthony, 299, 300

Goedeken, Edward A., article by, 180-208

Gold rush, California, 197, 270

Gondrecourt, France, 381, 404

Good Seeds: A Menominee Indian Food Memoir, reviewed, 354-55

Goosetown neighborhood, Iowa City, 310

Gorenfeld, Will, and John Gorenfeld, book by, reviewed, 341-42

Gorman, Kathleen, book reviews by, 349-50, 442-43

Gotch, Frank, 401

Gower and Holt store, 282

Grain Processing Corp., 58

Grange, Iowa, 53

Grant, H. Roger, book review by, 134-35

Grant, Ulysses S., 178

Grant, W. L., 171

Grassley, Charles, 36, 42

Greasley, Philip A., book edited by, reviewed, 433-35

The Great Exception: The New Deal and the Limits of American Politics, reviewed, 141-42

Great Plains Indians, reviewed, 335-36

Green, George, 266

Green, H. H., 276

Grenville Mellen Dodge in the Civil War: Union Spymaster, Railroad Builder and Organizer of the Fourth Iowa Volunteer Infantry, reviewed, 226-28

Griffith, John L., 397-401

Grimes, James, 4

Grimstead, Jacob, 39 
Griswold, Wendy, book by, reviewed, 451-53

Groggins, P. H., 57

Gubbels, Thomas, book review by, 137-39

Gubernatorial Stability in Iowa: A Stranglehold on Power, reviewed, 316-34

Gue, Benjamin, 181, 188-91, 192-96, 200, 205-7; photo of, 189

The Guerrilla Hunters: Irregular Conflicts during the Civil War, reviewed, 442-43

Gustafson, Robert K., 82

Gutiérrez, Aurelia, 421, 430

Gutiérrez, Juan, 421, 424, 430

Hadden, Thomas B., 82

Haddock, William, 288

Hadley, Emelia, 262

Halleck, Henry, 165-66, 169

Haney, Deanna R., book edited by, reviewed, 445-46

Hannon, Rev. Mathias, 300

Hansen, Fred W., 396

Hansen, Marcus Lee, 384-85, 388, 395

Harden, Patrick, 96

Harding, William L., 313

Harrington, Jerry, article by, 1-46; book by, reviewed, 453-55

Harris, William C., book by, reviewed, 439-40

Hart, David, 78, 100-101, 104, 105

Harvest of Hazards: Family Farming, Accidents, and Expertise in the Corn Belt, 1940-1975, reviewed, 456-57

Hawkins, Lex, 21

Hawley, Marlin F., book review by, 217-20

Heacock, C. C., 281

Heideman, Eugene P., book by, reviewed, 222-24

Heinz, Anne M., and John P. Heinz, book edited by, reviewed, 119-21

Helena, Arkansas, 164-74, 178-79

Helstein, Ralph, 103

Hendrik P. Scholte: His Legacy in the Netherlands and in America, reviewed, 222-24

Henry, Ben, 79, 82, 86, 90, 92, 95
Henry, Lyell D., Jr., book by, reviewed, 137-39

Herbert Hoover in the White House: The Ordeal of the Presidency, reviewed, 363-66

Herbert Hoover: A Life, reviewed, 363-66

Hercules Manufacturing Plant, 59

Herring, Clyde, 57

Hickenlooper, Bourke, 62

Hidden Thunder: Rock Art of the Upper Midwest, reviewed, 337-38

Hill, Eugene M., 39

Hill, Lena M., and Michael D. Hill, book edited by, reviewed, 457-59

Hilton, Iowa, 29

Hinojosa, Felipe, book review by, 252-54

History of Transportation, Henry County, Iowa, reviewed, 134-35

Hobbie, Charles, 86, 90

Hoegh, Leo, 103, 104, 107

Hoehnle, Peter: book by, reviewed, 245-46; book review by, 343-45

Hokanson, Drake, book review by, 254-56

"Home Away from Home: The Camp Dodger Newspaper and the Promotion of Troop Morale, 1917-1919," 381-405

Hooper, Candice Shy, book by, reviewed, 232-33

Hoover, Henry, 78-79

Hopper, Alfred, 174

Horses, 173

Hostess houses, World War I, 393

Housing, for Mexican railroad workers, 417-19, 423-26

Howes, Edward S., 386, 387

Hubbard, Charles M., book review by, $439-40$

Hughes, Harold, 1-2, 10-46, 73, 10910, 111; photos of, 12, 33

Hultman, Evan, 24-25, 28

Hussan, Yussif, 402

Immigrants and immigration, 412, 415, 417; Mexican, 406-32; Scandinavian, 162, 167, 172, 176. See also Ethnicity and individual ethnic groups 
Independence, Iowa, 67-68

Independence Canning Corp., 67-68

Industrial and Defense Commission, Iowa, 50, 55-62

Industrial development, 47-70

Industrial Resources and Defense Council, Iowa, 53-55

Industrial Union Council: Cedar Rapids, 82; Iowa-Nebraska States, 82, 83, 85; Ottumwa, 78-81, 84, 92, 96

Insurance, auto, 35

Insurgent Democracy: The Nonpartisan League in North American Politics, reviewed, 139-41

Invisible Hawkeyes: African Americans at the University of Iowa during the Long Civil Rights Era, reviewed, 457-59

Iowa . . . Land of Industrial Opportunity, 64-67

Iowa Agricultural College and Model Farm. See Iowa State University

Iowa City, Iowa, 282, 283, 290-315, 406-32

Iowa City Daily Citizen, 415-16, 41921

Iowa Federation of Labor (IFL), AFL-CIO, 53, 104-5, 108, 109, 111

Iowa Poll, 9

Iowa State College. See Iowa State University

Iowa State University, 56, 58, 63-64, 180-208; photo of campus of, 205

“Iowa's Last Liquor Battle: Governor Harold E. Hughes and the Liquorby-the-Drink Conflict," 1-46

Ioway Life: Reservation and Reform, 1837-1860, reviewed, 118-19

Irish Catholics, 299, 300, 301, 302, 304, 307, 308-10

The Iron Road in the Prairie State: The Story of Illinois Railroading, reviewed, 135-37

Ironstrack, George, book review by, 435-37

Jack, Zachary Michael, book by, reviewed, 463-64
Jeansonne, Glen, book by, reviewed, 363-66

The Jefferson Highway: Blazing the Way from Winnipeg to New Orleans, reviewed, 137-39

Jellison, Katherine, book review by, 456-57

Jenkins, Thomas H., 388

Jennings, Chris, book by, reviewed, 343-45

Jensen, Dwight, 28-29

Jews, 35

Jews in Wisconsin, reviewed, 352-54

John Deere and Co., 53, 78, 92

John Morrell and Co., 71, 73-75, 77$86,98,99,100$

Johnston, Matthew N., book by, reviewed, 239-41

Johnston, Robert D., book review by, 224-26

Johnston, Iowa, 381-405

Jones, Donald, 79, 83, 85, 98-99

Jones, George, 204

Jonesville Union School, 197

Journalism, 381-405

Jung, Patrick J., book review by, 33940

Kane, Randy, book review by, 236-38

Kane, William W., 396

Kanter, Jodi, book by, reviewed, 366-68

Kearny's Dragoons Out West: The Birth of the U.S. Cavalry, reviewed, 341-42

Kehrberg, Richard F., book review by, 226-28

Keokuk, Iowa, 58, 280; sketch of, 280

Key clubs, 7, 43

Kimball, Edward, 53, 57, 59, 61, 62

Kinsey, Joni, book review by, 239-41

Kirkwood, Samuel, 191

Klein, Allen, 53, 55

Klein, James E., book review by, 126-27

Klobuchar, Amy, book by, reviewed, 462-63

Knights of Columbus, 393, 398

Knudsen, Otto, 57, 58

Koppel, A. M., 172

Krubil, Francis, 301

Kublik, Leo, 30 
Labor unions, 71-113

Land-grant movement, 182-87

Land markets, 263-64, 266-68

Land Too Good for Indians: Northern Indian Removal, reviewed, 220-22

"'Land Was the Main Basis for Business': Markets, Merchants, and Communities in Frontier Iowa," 261-89

Lane, Carroll, 40

Lansing, Michael, book by, reviewed, 139-41

Larew, James C., review essay by, 316-27

Larimer, Christopher W., book by, reviewed, 316-34

Larsen, Paul, 106, 112

Last Days of Red Cloud Agency: Peter T. Buckley's Photograph Collection, 1876-1877, reviewed, 236-38

Latino Heartland: Of Borders and Belonging in the Midwest, reviewed, 252-54

Latta, Robert Ray, 274-75

Lauck, Jon K., book review by, 243-45

Lause, Mark A., book by, reviewed, 437-39

Lawrence, Edna, 97, 98

League of Women Voters, 109

Lear, Martin, 28

Lee, Joseph, 391-92

Lee, R. Alton, book by, reviewed, 449-51

Leslie, Thomas, book review by, 149-50

Lewis, Ed "Strangler," 402

Lincoln, Abraham, 164, 181, 186

Lincoln and Congress, reviewed, 439-40

Lincoln and Emancipation, reviewed, 230-32

Lincoln and the Democrats: The Politics of Opposition in the Civil War, reviewed, 440-41

Lincoln and the Thirteenth Amendment, reviewed, 230-32

Lincoln's Generals' Wives: Four Women Who Influenced the Civil War - for Better and for Worse, reviewed, 23233

Lindaman, Matthew, article by, 381405
Liquor Control Commission, Iowa, $6-7,27,39,43,44-45$

Liquor legislation, 1-46

"Local 1's Unionism and the Transformation of Iowa's Politics, 19391970," 71-113

Loghry, Ermol, 15

Long, Irving, 40

Lookingbill, Brad D., book review by, 114-15

Loras, Bishop Mathias, 295-98; sketch of, 297

Lost Buxton, reviewed, 448-49

Loveland, Albert, 93-94

Loveless, Herschel, 73, 78, 96-101, 107-9

Lucas, W. V., 274

Maffitt, Dale L., 53

Magliari, Michael F., book review by, 139-41

Mahoney, Timothy R., book by, reviewed, 224-26

Main, Franklin, 43-44

"The Making of a Midwestern Catholicism: Identities, Ethnicity, and Catholic Culture in Iowa City, 1840-1940," 290-315

Manhood on the Line: Working-Class Masculinities in the American Heartland, reviewed, 246-48

Manns, Ernest, 80

Manufacturers Association, Iowa, 53, 102, 107, 109

Margis, Matthew J., book review by, 132-34

Marion, Iowa, 279

Markets, 261-89

Marsh, James, 275

Mart, Michelle, book by, reviewed, 248-49

Martin, Lawrence H., 404

May, George, 69

May, Louis C., 83

Mazzuchelli, Fr. Samuel, 295-98; sketch of, 296

McCann, Dennis, book by, reviewed, 443-45

McCormick, Rev. J. P., 300 
McCoy, Jack, 78, 92, 100-101, 102, 104, 105, 109

McDowell, Irwin, 169

McKerley, John W., book review by, 234-35

McKnight, Brian D., and Barton A. Myers, book edited by, reviewed, 442-43

McMahon, David R., book review by, 152-53

McReynolds, Wade, 83, 101

Meagher, John, 97, 98

Means Grocery, Iowa City, 428

Medford, Edna Greene, book by, reviewed, 230-32

Melendy, Peter, 181, 190-96, 200; photo of, 192

Merchants, 279-83

Merrill, Iowa, 29

Methodists, 34, 36, 300

Mexican Americans, 406-32

Meyer, Louis, 26

Meyer, Sabine N., book by, reviewed, 126-27

Meyer, Stephen, book by, reviewed, 246-48

Michels, Fr. Mathias, 301-2, 305

Michigan Agricultural College, 193, 202

Michigan State Normal College, 197

"Midwestern Writers on Environmental Stewardship: A Review Essay," 209-15

Migration, rural-to-urban, 48-50, 54, 64,65

Military history, 161-79

Miller, Charlie, 431

Miller, Frank, cartoon by, 20

Miller, Jay, book by, reviewed, 217-20

Mills, George, 8, 37

Mills, Ray, 104

Mincks, Jacob “Jake," 78, 91-92, 94, 100-105, 108, 110-11

Mississippi River, 281

Missouri Law and the American Conscience: Historical Rights and Wrongs, reviewed, 234-35

Mitchell, Jack, book by, reviewed, 455-56

Mitchell, Robert B., book review by, $445-46$
Moody, William, 40

Moon, Edwin, 5

Moon Law, 5-6

Moore, Shirley Ann Wilson, book by, reviewed, 345-47

Mooty, William, 110

Morain, Tom, book review by, 464-65

Morgans, James Patrick, book by, reviewed, 226-28

Morrill, Justin, 181, 185-87, 194-95

Morrill Land-Grant Act, 181-82, 185-87

Morse, Sarah, 277

The Most Famous Writer Who Ever Lived: A True Story of My Family, reviewed, 370-72

Motor Truck Association, Iowa, 10

Mowry, John, 41

Mulct Law, 5-6

Murphy, J. T., book reviews by, 238 $39,345-47$

Murray, John, 44

Murray, Margaret Archer, 273, 274

Murray, William, 63

Muscatine, Iowa, 26, 58

Music, 394-96

Mutel, Cornelia F., book by, reviewed, 209-15

Myers, Barton A. See McKnight, Brian D.

Nagle, George, 23, 32

Naick, Patrick, book review by, 45759

Narrating the Landscape: Print Culture and American Expansion in the Nineteenth Century, reviewed, 239-41

Nash, Louis, 391

National Defense, Council of, 57

National Farmers Organization (NFO), 105

Neely, Mark E., Jr., book by, reviewed, 440-41

Nelson, Henry C., 22

Never Curse the Rain: A Farm Boy's Reflections on Water, reviewed, 459-60

Nevin, David, 96, 98

New Deal, 49

A New Deal for South Dakota: Drought, Depression, and Relief, 1920-1941, reviewed, 449-51 
Newlin, Keith, book edited by, reviewed, 129-31

The News from Lone Rock: Observations and Witticisms of a Small-Town Newsman, reviewed, 445-46

Newspapers, 381-405

Nichols, Jeff, book review by, 453-55

Nilson, Carl, 79

Ninth Iowa Infantry Regiment, 161

Noe, Marcia, book review by, 370-72

Nunnally, Patrick, book review by, 443-45

Nye, Frank, 20, 41, 43

O'Brien, R. R., 53

O'Farrell, Fr. John, 310

O'Malley, George, 41

Oden, Derek S., book by, reviewed, 456-57

Olson, Daron W., book review by, 446-48

Olson, Greg, book by, reviewed, 118-19

Ordnance plants: Iowa (near Burlington), 59; Des Moines, 59

Orejel, Keith, article by, 47-70

"The Origins of the Iowa Development Commission: Agricultural Transformation and Industrial Development in Mid-TwentiethCentury Iowa," 47-70

Orwig, Darrek D., book edited by, reviewed, 132-34

Ossian, Conrad, 41

Otjen, Nathaniel, article by, 406-32

Otto, Joseph, book review by, 459-60

Ottumwa, Iowa, 71-113

Øverland, Orm, book edited by, reviewed, 446-48

The Oxford Handbook of American Immigration and Ethnicity, reviewed, 250-52

Panic of 1837, 273

Panic of 1857, 268, 276

Paradise Now: The Story of American Utopianism, reviewed, 343-45

Parcell, Lester, 96

Parsons, Mary Jane, 276

Patterson, Robert, 57

Peak, Richard, 26
Pedrick, Frank, 83

Pelamourgues, Anthony, 299

Pesch, Carl, 25, 28, 29

Pesticides, A Love Story: America's Enduring Embrace of Dangerous Chemicals, reviewed, 248-49

The Pew and the Picket Line: Christianity and the American Working Class, reviewed, 143-44

Pfeifer, Michael J., article by, 290-315

Phillips, Christopher, book by, reviewed, 121-24

Pickford, Arthur, 285

Pitzer, Robert, 270

Planning Democracy: Agrarian Intellectuals and the Intended New Deal, reviewed, 243-45

Plowed Under: Food Policy Protests and Performance in New Deal America, reviewed, 143-44

Plummer, Gen. Edward H., 388

Policy Committee, Ottumwa, 96

Political Action Committee (PAC), $\mathrm{CIO}, 83,86,92-95,102$

Ponce, Guadalupe, 422

Ponce, Selso, 422

Post War Rehabilitation Commission, Iowa, 61

Potter, James E., book edited by, reviewed, 236-38

Poyet, B. M., 299, 300-301

Prairie Visions: Writings by Hamlin Garland, reviewed, 129-31

Preferred Risk Mutual Insurance Co., 35

Presidential Libraries as Performance: Curating American Character from Herbert Hoover to George W. Bush, reviewed, 366-68

Pridmore, Jay, book by, reviewed, 149-50

Pritchard, James A., book review by, 372-74

Progressive Party, 86

Prohibition. See temperance and prohibition

Provenzano, James, 82

Public Safety, Iowa Department of, 25

Putz, Paul Emory, book review by, 143-44 
Quartermasters, Civil War, 161-79

Railroads, 277-78, 283, 406-32

Ramírez, Isabel, 423

Ramírez, Louis, 423, 424

Randolph Scott E., book review by, 135-37

Rankins, Peter, 30

Rappleye, Charles, book by, reviewed, 363-66

Rasmussen, Anders Bo, article by, 161-79

Rath Packing Co., 74, 82, 113

Raymond, Elizabeth, book reviews by, 129-31, 433-35

Reapportionment, legislative, 9, 32, 102, 109-10

Red Cross, 85

Register, Cheri, book by, reviewed, 209-15

Religion, 17-18, 34-36, 290-315, 402-3

Remsen, Iowa, 29

Republican Party, 10, 15, 22-23, 32, 37, 72, 75-76, 84, 188

Restaurant Association, Iowa, 15

Richardson, Robert A., 190

Richman, Irving B., 265, 274

Right-to-work law, 86-87, 102, 104

Rigler, Robert, 38

Riley, Tom, 34

Rivers, 275-76, 281, 283, 284

The Rivers Ran Backward: The Civil War and the Remaking of the American Middle Border, reviewed, 121-24

Robertson, Breanne, book review by, 146-48

Rock Island Magazine, 406-7, 411, 424-25

Rodríguez, Jesús, 415-16

Rogers, Wilson (Moose), 82

Roosevelt, Franklin D., 74, 77, 80, 8384

Rosenberg, Gabriel N., book by, reviewed, 361-63

Rosenthal, Robert, 27

Rowland, L. A., 53, 54

Rural-to-urban migration, 48-50, 54, 64,65

Ryder, Lorenz, 402
The Sacred Cause of Union: Iowa in the Civil War, reviewed, 350-52

St. Agatha's Seminary, Iowa City, 309

St. Anthony's Catholic Church, Davenport, 299

St. Boniface League of Iowa, 313

St. Francis Xavier Catholic Church, Iowa City, 304-6

St. John, Mary, 288

St. Mary's Catholic Church, Iowa City, 290, 294, 297-304, 307-8, 311-15; photos of, 298, 313, 314

St. Patrick's Catholic Church, Iowa City, 309; photo of, 308

St. Paul the Apostle Catholic Church, Burlington, 296

St. Wenceslaus Catholic Church, Iowa City, 310; photo of, 311

Samito, Christian, book by, reviewed, 230-32

Sánchez, Angeleta, 423

Sánchez, José, 423, 424, 428

Sanders, W. E., 277

Scandinavian immigrants, 162, 167, 172,176

Schaefer, Herman, 96

Schantz, Mark S., book review by, 437-39

Schell, James Peery, 270

Scherle, Bill, 42

Schrab, Geri, and Robert F. Boszhardt, book by, reviewed, 337-38

Schroeder, Jack, 33-34, 37, 39

Schulte, A. J., 312-14

Schwantes, Carlos A., book review by, 128-29

Science education, 184

Scott, Rachel, 266

Seaba, Henry, 265

Sears, Charles, 80

Seeman, Corey, book review by, 460-61

Selby, Rodney Q., 56, 57, 61, 62

The Selected Letters of Laura Ingalls Wilder, reviewed, 131-32

The Senator Next Door: A Memoir from the Heartland, reviewed, 462-63

Shaff, David O., 32, 39, 109

Shaff Plan, 32, 109, 110

Shannon, Archie, 67 
Shroder, Tom, book by, reviewed, 370-72

Siddali, Silvana, book by, reviewed, 115-18

Silvis, IL, 406, 408, 421, 425

Simon, Lee, 88, 90, 91

Singing, 394-96

Sioux City, Iowa, 27-28, 283

Sioux City Journal, 22

Sipma, Sjoerd Aukes, 269

Sisters of Charity of the Blessed Virgin Mary, 304, 309

Slaves and slavery, 164-65, 178-79

The Small-Town Midwest: Resilience and Hope in the Twenty-First Century, reviewed, 254-56

Smith, Bishop Gordon, 34-35

Smith, Ralph, 53

Smyth, Bishop Clement, 304

Solonick, Justin S., book by, reviewed, 228-29

Somewhere Over There: The Letters, Diary, and Artwork of a World War I Corporal, reviewed, 132-34

Spaulding, Walter R., 396

"The Spoils of the Victors: Captain Ferdinand Winslow and the 1863 Curtis Court of Inquiry," 161-79

Sprcek, Adolph, 304

Staley, Oren Lee, 105

Standfield, Sophia, 265, 288

Stanton, Edwin, 164

Stars and Stripes, 382

State University of Iowa. See University of Iowa

Steamboats, 276, 281, 283, 284

Steele, Frederick, 167-68, 171

Stek, Pam, book review by, 448-49

Stewart, Catherine, book review by, 150-52

Stewart, Ruth, 386, 390-91, 395

Stock, Catherine McNicol, book review by, 449-51

Stone, William, 189, 190

Stores, 277, 279-83

Stover, Fred W., 87, 88, 90

Street, Warren R., book edited by, reviewed, 238-39

Street, William D., book by, reviewed, 238-39
The Suburban Church: Modernism and Community in Postwar America, reviewed, 368-70

Sueppel, John, 307

A Sugar Creek Chronicle: Observing Climate Change from a Midwestern Woodland, reviewed, 209-15

Sutliff, A. C., 264

Svendsen, Gro, 270, 288

Swaney, Mary Ann, 273

Sweet Freedom's Plains: African Americans on the Overland Trails, 18411869, reviewed, 345-47

Swisher, Scott, 36

Tackett, Michael, book by, reviewed, 460-61

Taft-Hartley Act, 86

Teamsters, International Brotherhood of, 79

Technical colleges, 183

Telfer, Rev. John Harley, 88-89

Temperance and prohibition, 3-7

Temperance League, Iowa, 17

Temperance Legislative Council, 17

This Storied River: Legend E Lore of the Upper Mississippi, reviewed, 443-45

Thomas Hart Benton: Discoveries and Interpretations, reviewed, 146-48

Thomas, Ben, 27

Tobacco use, 393

Torgerson, Julian, 27-28

Townsend, Norton S., 204

Tractors, 49, 60

Trades and Labor Assembly, Ottumwa, 78-79, 96

Traqueros, 406-32

Trombly, Louis, 266-67

Tronnes, Libby, book review by, 220-22

Turner, Jonathan, 185

Twenty-First Amendment to the U.S. Constitution, 6

Twenty-five Years among the Indians and Buffalo: A Frontier Memoir, reviewed, 238-39

Tyson, Robert, 38

Unions, labor, 71-113

United Auto Workers, 78, 92 
United Farm Equipment and Metal Workers Union (FE), 86, 91, 92

United Mine Workers of America, Iowa, 53

United Packinghouse Workers of America, 71-113

University of Illinois, 399

University of Iowa, 10, 13, 207, 385

Vail, David D., book review by, 24849

Vandever, William, 161-63

Van Eaton, Charles, 41

Van Nuys, Frank, book by, reviewed, 357-58

Van Tassel, Walter, 98-99

Van Zetten, John, 67

The Vanishing Messiah: The Life and Resurrections of Francis Schlatter, reviewed, 355-57

Varmints and Victims: Predator Control in the American West, reviewed, 357-58

Vázquez, Salvador, 416

Vega, Sujey, book by, reviewed, 25254

Venereal disease, 384

Villafán, Rafael, 412-14, 419

Wages, for Mexican railroad workers, 430

Wakeley, Ray, 63

Walch, Timothy, book review by, 366-68

Walker, John A., 39

Wallace, Henry A., 57, 87, 90

Walsh, Marie F., 424-25

Wapello County, 71-77, 80, 83-84, 94, 108

War Manpower Commission, Ottumwa, 84

The War of 1812 in Wisconsin: The Battle for Prairie du Chien, reviewed, 339-40

Warner, Thomas, 262

Warren, Wilson J., article by, 71-113

Washburne, C. C., 170

Water transport, 275-76, 281, 283, 284

Waterloo, Iowa, 27, 74, 82, 85, 104, 106, 112-13
We Are What We Drink: The Temperance Battle in Minnesota, reviewed, 12627

Weber, Jennifer, book review by, 440-41

Webster, Francis H., book by, reviewed, 132-34

Weingar, Irving A., Rabbi, 35

Weitzel, David N., book by, reviewed, 355-57

Welch, Adonijah, 181, 196-208

Weninger, Franz Szvier, 301, 304, 305

Wentling, Sonja, book review by, 352-54

Weso, Thomas Pecore, book by, reviewed, 354-55

West Liberty, Iowa, 411, 422

White, Andrew, 193

White, Ann Folino, book by, reviewed, 143-44

Whitney, Harold, 79

Whittaker, William, book review by, 341-42

Why Iowa Is Great, 67

Wilson, Frank, 53, 54-55

Wilson, George A., 53-57, 61

Winn, Kenneth H., book edited by, reviewed, 234-35

Winslow, Ferdinand, 161-79; photo of, 175

Wisconsin on the Air: 100 Years of Public Broadcasting in the State That Invented It, reviewed, 455-56

Wish You Were Here: Love E Longing in an American Heartland, reviewed, 463-65

Wishart, David J., book by, reviewed, 335-36

Wister, Owen, 394-95

Woman's Christian Temperance Union (WCTU), 4, 17

Women, in higher education, 206-7

Women, Work, and Worship in Lincoln's Country: The Dumville Family Letters, reviewed, 119-21

Wood, W. Raymond, book review by, 335-36

Woodrow, Jack, 79

Workshops of Empire: Stegner, Engle and American Creative Writing during the Cold War, reviewed, 150-52 
World War I, 313-14, 381-405, 410, 417

World War II, 49, 50, 52-62, 84-85

Wrestling, 401-2

A Wrestling Life: The Inspiring Stories of Dan Gable, reviewed, 152-53

Wright, Ed, 190

Wright, William, store, 283

Wynn, William, 207
Xanten, John, 307

Yoder, Franklin, book review by, 124-26

Young Women's Christian Association (YWCA), 393

Young, Sam, 272

Zbyszko, Wladek, 402

Zwinge, Capistran, 304-6 


\section{Montana The Magazine of}

Western History

For 65 years ... one of the best history magazines in the nation! Contact us for subscriptions, back issues, advertising opportunities, and to ask about the books we publish. Mention this ad to receive a free gift with your new subscription.

Big Sky. Big Land. Big History. Montana Historical Society montanahistoricalsociety.org tryan@mt.gov (406)444-4708

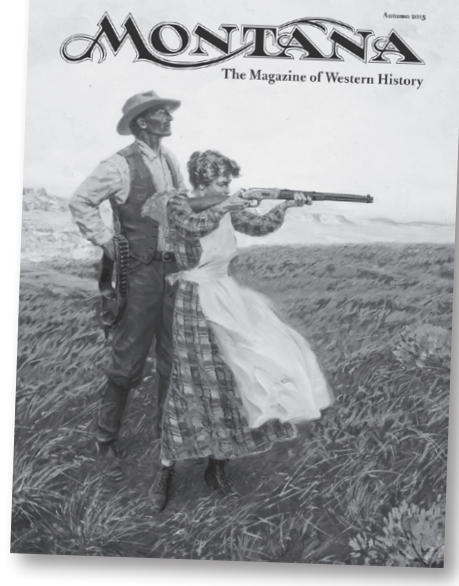
225 N. Roberts, P.O. Box 201201 Helena, MT 59620-1201 


\section{Contributors}

MATTHEW LINDAMAN is professor of history and chair of the Department of History and Legal Studies at Winona State University, where he regularly teaches courses on World Wars I and II. He earned his M.A. in history from the University of Northern Iowa and Ph.D. in history from the University of Kansas. He is working on a book analyzing John L. Griffith's role in intercollegiate athletics between the wars, including serving as the first commissioner of the Big Ten Conference.

NATHANIEL OTJEN is a doctoral student in Environmental Sciences, Studies, and Policy (focal department: English) at the University of Oregon. He holds a B.A. in English and Anthropology from the University of Iowa. He studies contemporary environmental literature, and his research aims to incorporate literary criticism into multispecies studies. 


\section{The State Historical Society of lowa}

The Annals of Iowa is published quarterly by the State Historical Society of Iowa, the Historical Division of the Department of Cultural Affairs of the State of Iowa. The society operates from two centers, Des Moines and Iowa City. A museum, research library, state archives, special collections, community programming, historic preservation, and membership programs are located at 600 East Locust Street, Des Moines, IA 50319, phone 515-281-5111. Publications, a research library, and special collections are located at 402 Iowa Avenue, Iowa City, IA 52240, phone 319-335-3916. The society also operates several historic sites across the state.

\section{Subscriptions}

Subscriptions to The Annals of Iowa are $\$ 24.95$ per year; single copies are $\$ 7$. Contact Publications, State Historical Society of Iowa, 402 Iowa Avenue, Iowa City, Iowa 52240.

The Annals is available on microfilm from Xerox University Microfilms, $300 \mathrm{~N}$. Zeeb Road, Ann Arbor, Michigan 48106.

\section{Submissions}

The Annals of Iowa invites the submission of articles on Iowa history and on subjects concerning the nation and the Midwest with an Iowa focus. State, local, and regional studies of political, economic, social, cultural, intellectual, institutional, ethnic, religious, material culture, archeological, and architectural history are welcome. The Annals also reviews significant books on related topics. A detailed set of editorial guidelines is available on request. All correspondence concerning editorial matters should be addressed to:

Marvin Bergman, editor

The Annals of Iowa

State Historical Society of Iowa

402 Iowa Avenue

Iowa City IA 52240

The Annals of Iowa is a participating member of the Conference of Historical Journals.

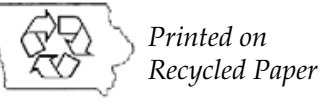

The acid-free paper used in this publication meets the minimum requirements of

(D) the American National Standard for Information Services-Permanence of Paper for Printed Library Materials, ANSI Z739.48B1984. 